\title{
Non-planar Reslicing for Freehand 3D Ultrasound
}

\author{
Andrew Gee ${ }^{1}$, Richard Prager ${ }^{1}$, and Laurence Berman ${ }^{2}$ \\ 1 Department of Engineering, University of Cambridge, UK \\ ${ }^{2}$ Department of Radiology, University of Cambridge, UK
}

\begin{abstract}
Any-plane slicing is a visualisation technique common to many medical imaging modalities, including 3D ultrasound. The acquired data is resampled on a user-specified plane and rendered, usually after some interpolation, on a standard graphics display. In this paper, we motivate and develop non-planar reslicing for freehand 3D ultrasound: the data is resampled not on a flat plane, but a curved surface, which is then unrolled for display on a flat screen. We show how to implement non-planar reslicing in a sequential manner, so the reslice image can be constructed directly from the raw B-scans and positions, without going through an intermediate voxel array stage. Care is taken to ensure that distances measured along lines in the non-planar reslice image are the same as the distances measured along the corresponding curves in 3D space. The resulting tool has interesting clinical applications, made accessible through an effective Graphical User Interface (GUI), which allows the user to specify the required reslice surface rapidly and intuitively. The GUI, and some of the applications, are illustrated in this paper.
\end{abstract}

\section{Introduction}

Conventional diagnostic ultrasound imaging is performed with a hand-held probe which transmits ultrasound pulses into the body and receives the echoes. The magnitude and timing of the echoes are used to create a $2 \mathrm{D}$ greyscale image (B-scan) of a cross-section of the body in the scan plane.

Using a technique called freehand 3D ultrasound imaging $[9,13]$, it is possible to construct $3 \mathrm{D}$ data sets from a series of $2 \mathrm{D}$ B-scans — see Figure 1. A conventional 3D freehand examination can be broken into three stages: scanning, reconstruction and visualisation. Before scanning, some sort of position sensor is attached to the probe. This is typically the receiver of an electromagnetic position sensor $[1,2,5,6,7,8,10,11,14]$, as illustrated in Figure 1, although alternatives include acoustic spark gaps [3], mechanical arms [4] and optical sensors [15]. Measurements from the position sensor are used to determine the positions and orientations of the B-scans with respect to a fixed datum, usually the transmitter of the electromagnetic position sensor. In the next stage, the set of acquired B-scans and their relative positions are used to fill a regular voxel array. Finally, this voxel array is visualised using, for example, any-plane slicing, volume rendering or surface rendering (after segmentation). 


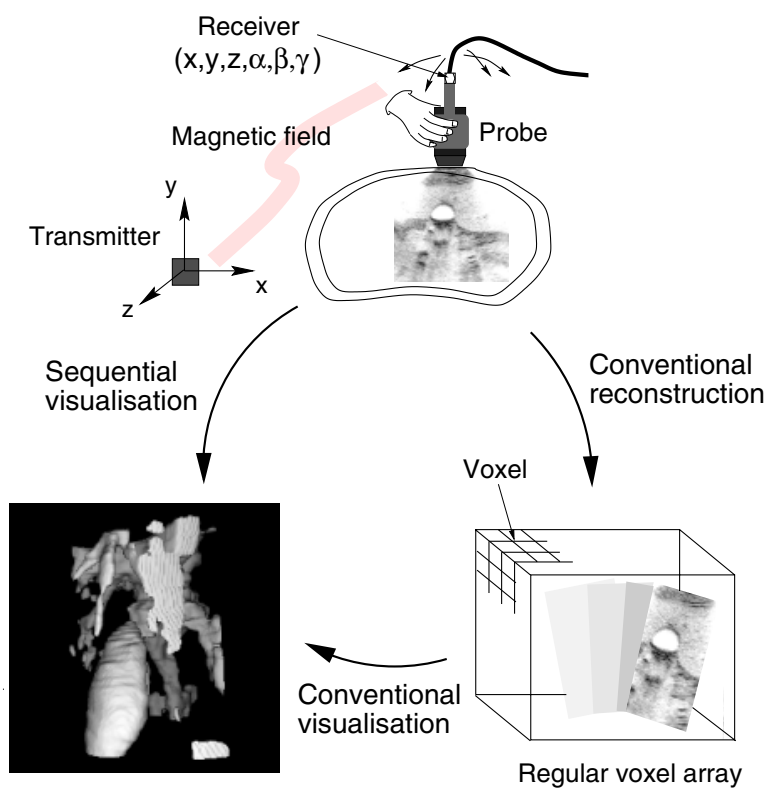

Fig. 1. Freehand 3D ultrasound imaging. The conventional approach is a three-stage process, involving scanning, reconstruction and visualisation. The alternative, sequential approach allows visualisation directly from the raw Bscans and positions. The figure illustrates an examination of a gall bladder.

Recently, we have proposed an alternative approach to freehand 3D ultrasound, which bypasses the voxel array stage. In sequential freehand 3D ultrasound, the data is visualised and analysed directly from the raw B-scans and positions - see Figure 1. Any-plane slicing, panoramic imaging, volume estimation and surface rendering can be performed without the use of an intermediate voxel representation $[5,6,7,14]$ : all of these facilities are implemented in the Stradx freehand 3D ultrasound system ${ }^{1}$. The sequential approach offers several advantages:

- When reslicing, the data is resampled only once, from the B-scan pixels to the slice pixels. The conventional approach requires two resampling stages, from the B-scan pixels to the voxel array, then from the voxel array to the slice pixels. Since resampling usually involves data approximation, more accurate visualisation is possible by avoiding one resampling process.

- Reslicing can be performed at the full resolution of the B-scan pixels without the significant memory overhead of a high resolution voxel array.

- Visualisation and data analysis can be performed in real time, as the data is being acquired, since the sequential visualisation and volume measurement

${ }^{1}$ Available at http://svr-www.eng.cam.ac.uk/ rwp/stradx/. 
algorithms reference each B-scan only once, in the order in which they are acquired.

- Segmentation (for volume measurement and surface rendering) is performed on the B-scans themselves, instead of parallel slices through the voxel array. The B-scans are high resolution and exhibit no reconstruction artifacts, making them relatively easy to interpret for manual or assisted segmentation. The same cannot be said of slices through the voxel array.

In this paper, we consider a visualisation technique called non-planar reslicing. This is similar to any-plane slicing, except the data is extracted along a simple curved surface instead of the usual flat plane. In Section 2, we describe how non-planar reslicing can be performed in the sequential framework, providing full implementation details. In Section 3, we show how the reslice surface can be specified using a simple, intuitive Graphical User Interface (GUI), such that the desired view is arrived at rapidly. In this section, we also illustrate several valuable in-vivo applications. Finally, we draw some conclusions in Section 4.

\section{Non-planar Reslicing}

Figure 2 illustrates the idea behind non-planar reslicing. A developable (unrollable) surface is specified by the user and its intersection with the $3 \mathrm{D}$ ultrasound data is computed, so that the surface can be 'painted' with the data it intersects. The painted surface is then unrolled for display on a flat screen.

The particular class of developable surface considered here is defined by a plane $\boldsymbol{\pi}$ with unit normal $\mathbf{n}$ and the plane curve $\mathbf{c}(s)$ lying in $\boldsymbol{\pi}$ - see Figure 2 . The surface is swept out by the set of rulings $\mathcal{R}$ which are all of length $2 r$, are all parallel to $\mathbf{n}$ and intersect $\mathbf{c}(s)$ at their midpoints. In the non-planar reslice image, we will refer to the unrolled plane curve and rulings as $\mathbf{c}^{\prime}(s)$ and $\mathcal{R}^{\prime}$ respectively.

In designing the reslicing algorithm, there are two issues of paramount importance:

1. Distances should be preserved, so that distances measured along lines in the non-planar reslice image are the same as the distances measured along the corresponding curves in 3D space.

2. The surface will intersect the B-scans along a set of curves, and some sort of interpolation will be required to produce a continuous reslice image. To be compatible with the sequential approach, the interpolation scheme should reference each B-scan only once, in the order in which they are acquired.

The starting point is the curve $\mathbf{c}(s)$ and the distance $r$, which are both specified by the user as described in Section 3. It is sensible to ensure that the scale of the reslice image (in $\mathrm{mm} / \mathrm{pixel}$ ) is the same as the scale of the original B-scans, so that the two types of image can be directly compared. The B-scan scaling can be deduced from the settings of the ultrasound machine or by a separate calibration process [8]. Using the same scaling, we can deduce the 


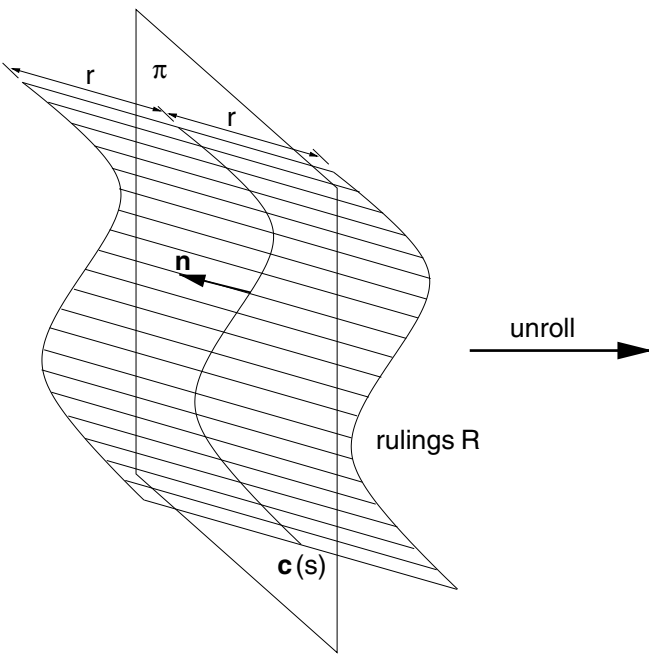

developable surface

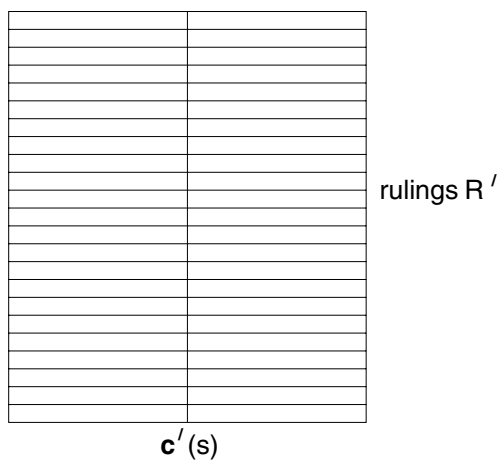

non-planar reslice image

Fig. 2. Non-planar reslicing. The non-planar reslice image shows the intersection of the $3 \mathrm{D}$ data with a special class of developable surface, unrolled for display on a flat screen.

length of the unrolled curve $\mathbf{c}^{\prime}(s)$ in pixels, which gives us the height of the reslice image. We can also use the scaling and $r$ to deduce the width of the reslice image in pixels.

The reslicing algorithm works with the set of rulings $\mathcal{R}^{\prime}$ passing through the pixel centres in the reslice image. Using the known scaling once more, we can deduce the corresponding rulings $\mathcal{R}$ in $3 \mathrm{D}$ space. We can also translate individual pixel centres in the reslice image to particular locations along the rulings $\mathcal{R}-$ see Figure 3. Having located each reslice pixel in 3D space, we can finally shade each pixel according to the intensity of the nearest B-scan pixel. It is desirable to introduce one user-defined parameter at this stage, namely the maximum distance $d$ allowable between the reslice pixel and the nearest B-scan pixel. In this way, areas of the surface which are a long way away from any recorded data will be left blank, instead of interpolated with misleading data.

Note that the line joining the reslice pixel to the nearest B-scan pixel will always be normal to the plane of the B-scan. This observation allows us to implement the interpolation scheme in an efficient, sequential manner. Consider the box defined by the two polygons a distance $d$ either side of the $n$th B-scan see Figure 4(a). This box defines the maximum range of influence of the pixels in B-scan $n$. Now consider the intersection of one of the rulings $\mathcal{R}$ with the box. Each extended B-scan pixel containing one of the reslice image pixel centres is a candidate for shading the reslice image pixel, since it is the closest pixel on 


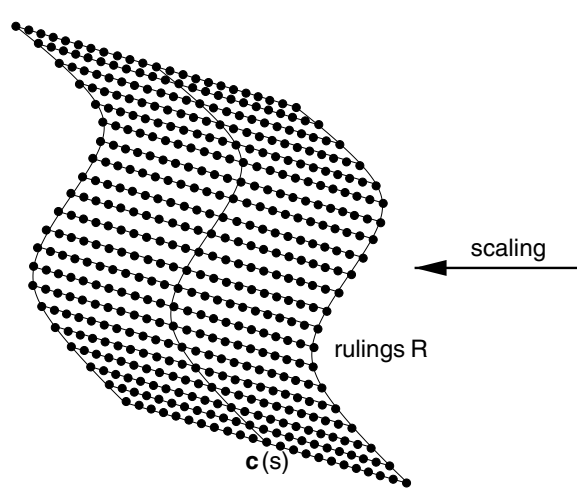

pixel centres in $3 D$ space

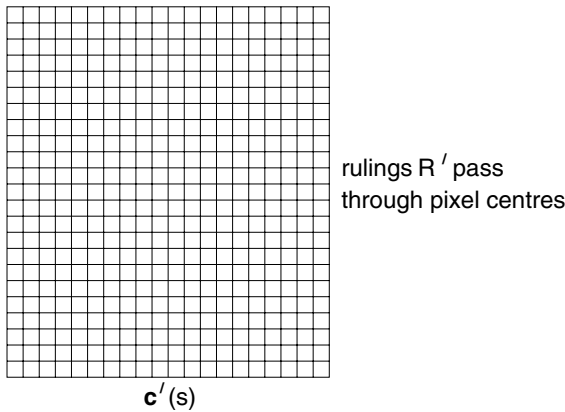

pixels in non-planar reslice image

Fig. 3. Pixel mapping. Using the known B-scan scaling, the centres of the individual pixels in the non-planar reslice image can be located in 3D space.

B-scan $n$ and lies within the distance limit $d$. The only circumstance that could change the shading of the reslice image pixel is if a pixel from another B-scan is closer. Therefore, we render the reslice image pixel with the intensity of the extended B-scan pixel it intersects, but at a depth $|z|$, where $|z|$ is the distance from the reslice image pixel centre to the B-scan plane. By exploiting the graphics z-buffer in this way, we are able to perform the interpolation efficiently in a sequential manner: should a pixel in a future B-scan be closer, the reslice image pixel will be rendered again at a shallower depth, overwriting the old value. The non-planar reslice image is therefore constructed as follows:

find rulings $R$ and 3D locations of reslice image pixel centres;

for each B-scan $n$

for each ruling in $R$

find any intersections of reslice image pixel centres with extended

B-scan pixels;

render the reslice image pixel with the intensity of the intersected B-scan pixel, at a depth $|z|$;

The fronto-parallel view in Figure 4(b) reveals how the intersection tests can be performed efficiently. For each ruling in $\mathcal{R}$, we locate only the first intersection $\left(x_{1}, y_{1}, z_{1}\right)$ to sub-pixel accuracy, and the increments $\Delta x, \Delta y$ and $\Delta z$. We then repeatedly add the increments to $\left(x_{1}, y_{1}, z_{1}\right)$ to find subsequent intersections, rounding down the $x$ and $y$ values to locate the intersected B-scan pixels.

Note that the interpolation scheme is well motivated by the physics of ultrasound acquisition. Since real ultrasound beams have a finite thickness, often as much as $10 \mathrm{~mm}$ [12], data in any particular B-scan is affected by structures a certain distance on either side of the B-scan plane. The notion of an extended 


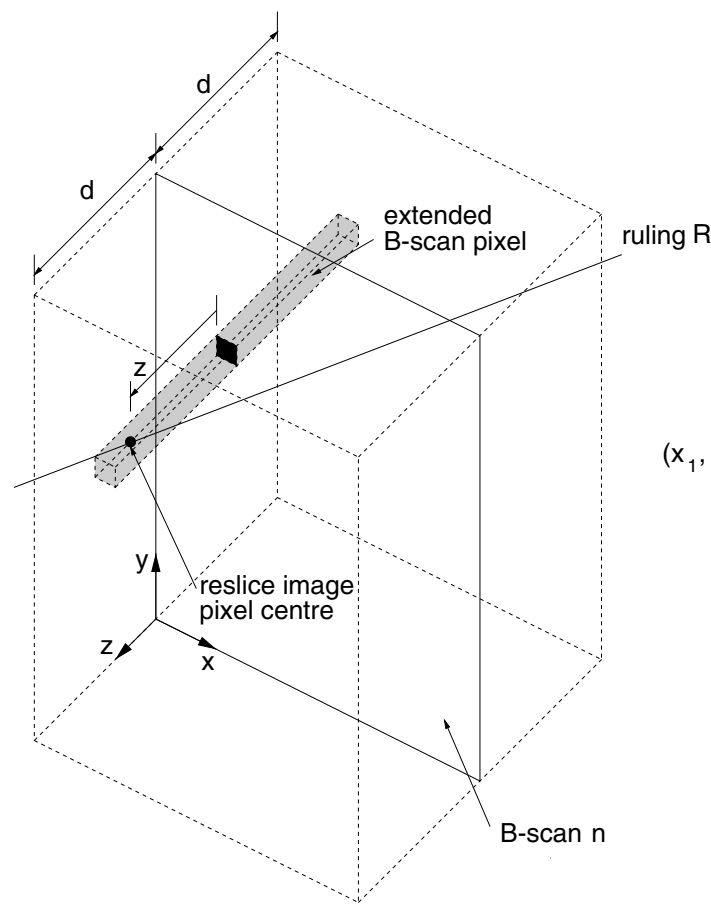

(a) oblique view

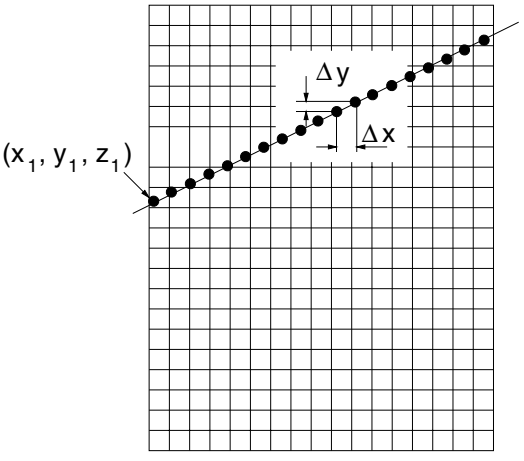

$\Delta z$ into the page

(b) fronto-parallel view

Fig. 4. Efficient sequential interpolation. The range of influence of B-scan $n$ is defined by a box extending a distance $d$ on either side of the B-scan, as shown in (a). Each reslice image pixel centre is tested for intersection with the box. If there is an intersection, the reslice image pixel is rendered with the intensity of the extended B-scan pixel it intersects, at a depth of $|z|$. In this way, the intensity will be updated if a subsequent B-scan lies closer to the reslice image pixel centre. The intersections can be calculated efficiently using an incremental algorithm, as shown in (b).

B-scan pixel, as defined in Figure 4, is therefore justified. Good practice would be to set the distance limit $d$ to half the width of the ultrasound beam.

\section{Defining the Reslice Surface}

If the non-planar reslice tool is to be useful, we need to develop a straightforward way for the user to specify the curve $\mathbf{c}(s)$ and the width $r$. In practice, $\mathbf{c}(s)$ will correspond to some curved anatomical structure that the user wishes to unroll.

The first stage is to find the plane $\boldsymbol{\pi}$ in which $\mathbf{c}(s)$ lies. This can be achieved using a standard any-plane slicing tool, such as the one already implemented in 
earlier versions of the Stradx freehand 3D ultrasound system $[5,6,7]$. The plane is defined by a line in one B-scan and a point in another, so that the resulting planar reslice reveals the key curved structure. The user can then draw along the structure to define $\mathbf{c}(s)$.

Consider the example in Figure 5, which illustrates an examination of a 16week foetus. The goal is to obtain a frontal view of the entire left leg, including all the toes: since the leg is bent at the knee and ankle, a non-planar reslice is necessary. The user starts by finding the plane $\boldsymbol{\pi}$ passing through the hip, knee and ankle. This is achieved by scanning through the B-scans until one is found which shows some of the fibula and tibia, as in Figure 5(a). The user draws a line down the middle of the two bones. To fully define the plane, the user now scans through the B-scans, following the femur up towards the hip, and marks a point near the top of the femur, as in Figure 5(b). The resulting planar reslice image, revealing the entire leg in profile, is shown in Figure 5(c). The user then draws along the leg to specify $\mathbf{c}(s)$. The non-planar reslice can now be constructed, using some default value of $r$, as in Figure $5(\mathrm{~d})$. The user can change $r$ by simply resizing the non-planar reslice window, while a slider at the bottom of the window controls the interpolation limit $d$. Note how all five toes are clearly visible in the non-planar reslice, ruling out one of the more common foetal abnormalities. Since lengths are preserved in the non-planar reslice, it is straightforward to measure the hip-to-toe distance along the unrolled leg, which is approximately $75 \mathrm{~mm}$.

A further application is illustrated in Figure 6: here, the goal is to construct a frontal view of a 22-week foetus' unrolled spine. The first step is to select a B-scan through the thorax, as in Figure 6(a), and draw a line between the vertebrae. The plane $\pi$ is fully defined by another point on the midline of the spine, marked by the user on a B-scan through the neck, as in Figure 6(b). The resulting planar reslice in Figure 6(c) shows the full length of the spine in profile. The user then draws along the spine to specify $\mathbf{c}(s)$. The non-planar reslice in Figure 6(d) shows a frontal view of the entire length of the unrolled spine, including some of the rib cage. The length of the spine, from neck to coccyx, is approximately $117 \mathrm{~mm}$.

\section{Conclusions}

The non-planar reslice tool is a valuable addition to the portfolio of visualisation techniques for freehand 3D ultrasound. It can be implemented in a sequential manner, allowing reslices to be constructed without the need for an intermediate voxel array. The sequential implementation described in this paper takes care to preserve distances, so the clinician can easily measure the lengths of curved anatomical structures. Two interesting applications in obstetrics have been illustrated. There are many other potential applications: the body is full of curved structures that would benefit from being visualised as a whole. 


\section{References}

1. P. R. Detmer, G. Bashein, T. Hodges, K. W. Beach, E. P. Filer, D. H. Burns, and D.E. Strandness Jr. 3D ultrasonic image feature localization based on magnetic scanhead tracking: in vitro calibration and validation. Ultrasound in Medicine and Biology, 20(9):923-936, 1994. 716

2. S. W. Hughes, T. J. D'Arcy, D. J. Maxwell, W. Chiu, A. Milner, J. E. Saunders, and R. J. Sheppard. Volume estimation from multiplanar 2D ultrasound images using a remote electromagnetic position and orientation sensor. Ultrasound in Medicine and Biology, 22(5):561-572, 1996. 716

3. D. L. King, D. L. King Jr., and M. Y. Shao. Evaluation of in vitro measurement accuracy of a three-dimensional ultrasound scanner. Journal of Ultrasound in Medicine, 10:77-82, 1991. 716

4. R. Ohbuchi, D. Chen, and H. Fuchs. Incremental volume reconstruction and rendering for 3D ultrasound imaging. In R. A. Robb, editor, Proceedings of Visualization in Biomedical Computing, SPIE 1808, pages 312-323. International Society of Optical Engineering, Bellingham, WA, USA, 1992. 716

5. R. W. Prager, A. H. Gee, and L. Berman. 3D ultrasound without voxels. In Proceedings of Medical Image Understanding and Analysis, pages 93-96, Leeds, UK, 1998. 716, 717, 722

6. R. W. Prager, A. H. Gee, and L. Berman. Real-time tools for freehand 3D ultrasound. In Medical Image Computing and Computer-Assisted Intervention MICCAI'98, pages 1016-1023, Cambridge, MA, USA, 1998. LNCS 1496, Springer. 716, 717, 722

7. R. W. Prager, A. H. Gee, and L. Berman. Stradx: real-time acquisition and visualization of freehand three-dimensional ultrasound. Medical Image Analysis, 3(2):129-140, 1999. 716, 717, 722

8. R. W. Prager, R. N. Rohling, A. H. Gee, and L. Berman. Rapid calibration for 3D freehand ultrasound. Ultrasound in Medicine and Biology, 24(6):855-869, 1998. 716,718

9. R. N. Rankin, A. Fenster, D. B. Downey, P. L. Munk, M. F. Levin, and A. D. Vellet. Three-dimensional sonographic reconstruction: techniques and diagnostic applications. American Journal of Roentgenology, 161(4):695-702, 1993. 716

10. R. N. Rohling, A. H. Gee, and L. Berman. Three-dimensional spatial compounding of ultrasound images. Medical Image Analysis, 1(3):177-193, 1997. 716

11. R. N. Rohling, A. H. Gee, and L. Berman. Automatic registration of 3-D ultrasound images. Ultrasound in Medicine and Biology, 24(6):841-854, 1998. 716

12. M. L. Skolnick. Estimation of ultrasound beam width in the elevation (section thickness) plane. Radiology, 180(1):286-288, 1991. 720

13. H. Steiner, A. Staudach, D. Spitzer, and H. Schaffer. Three-dimensional ultrasound in obstetrics and gynaecology: technique, possibilities and limitations. Human Reproduction, 9(9):1773-1778, 1994. 716

14. G. M. Treece, R. W. Prager, A. H. Gee, and L. Berman. Fast surface and volume estimation from non-parallel cross-sections, for freehand 3-D ultrasound. Medical Image Analysis, 3(2):141-173, 1999. 716, 717

15. J. W. Trobaugh, D. J. Trobaugh, and W. D. Richard. Three-dimensional imaging with stereotactic ultrasonography. Computerized Medical Imaging and Graphics, 18(5):315-323, 1994. 716 


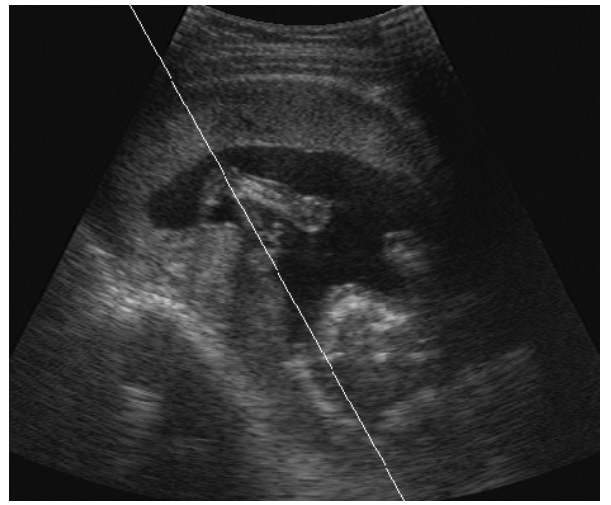

(a)

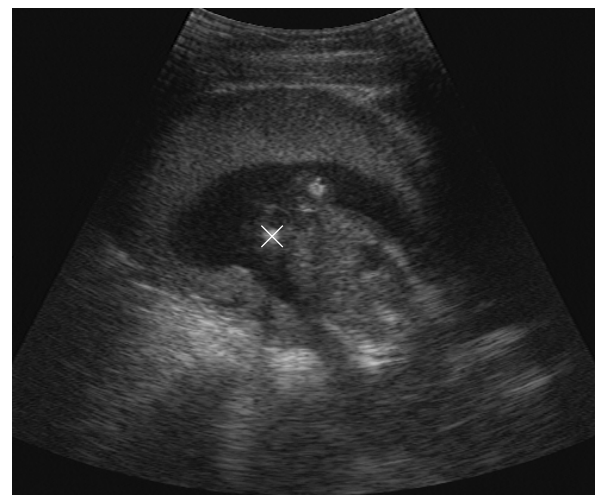

(b)

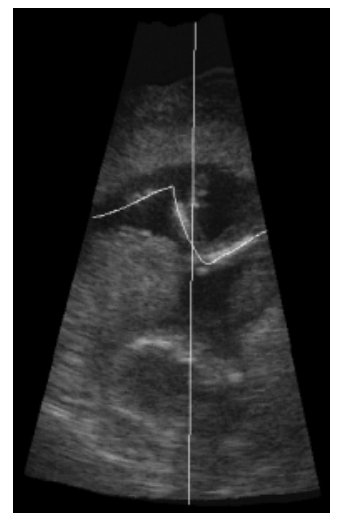

(c)

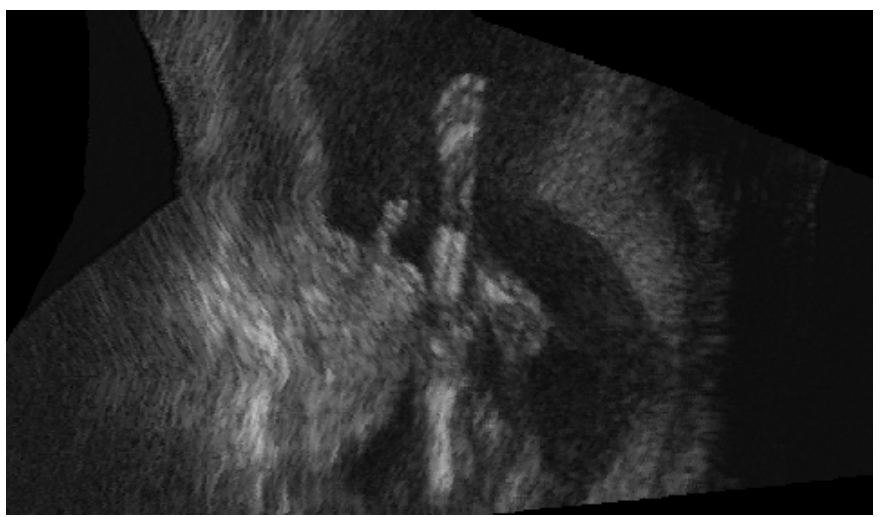

(d)

Fig. 5. Visualisation of a 16-week foetus' left leg. (a) and (b) show two B-scans from the recorded sequence. (c) is a planar reslice of the data, passing through the diagonal line in image (a) and the cross in (b). The planar reslice (c) shows the foetus' left leg in profile: the vertical line corresponds to the B-scan (a). (d) is a non-planar reslice, defined by the curve drawn by the user in (c). The non-planar reslice shows an unrolled, frontal view of the leg, including all five toes. 


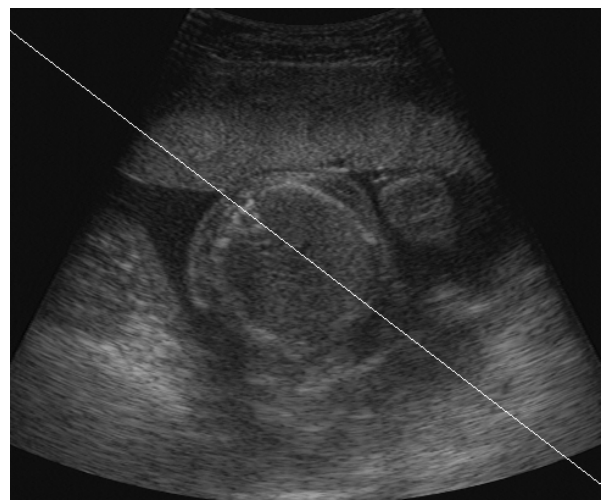

(a)

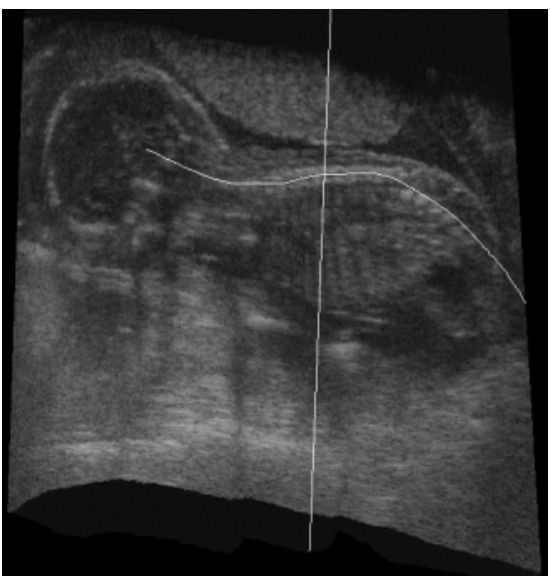

(c)

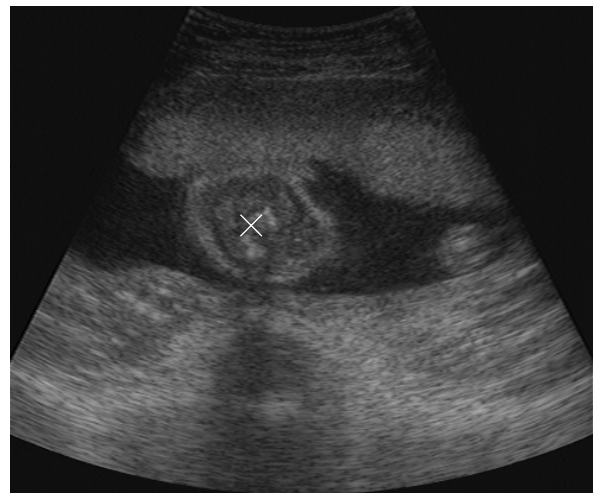

(b)

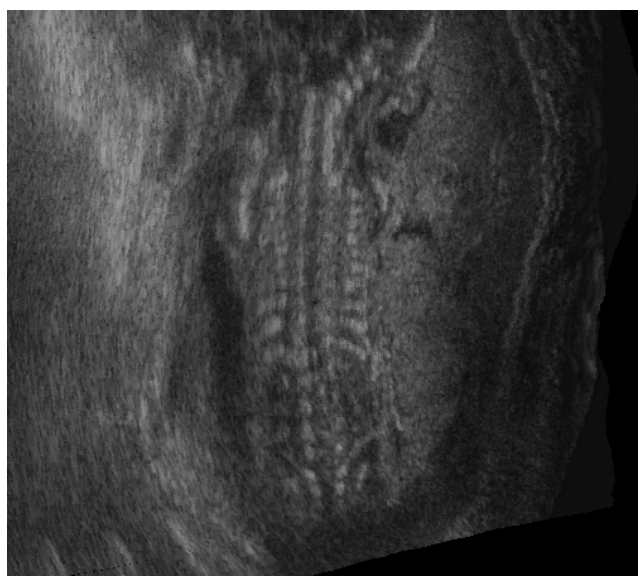

(d)

Fig. 6. Visualisation of a 22-week foetus' spine. (a) and (b) show two Bscans from the recorded sequence. (c) is a planar reslice of the data, passing through the diagonal line in image (a) and the cross in (b). The planar reslice (c) shows the foetus' spine in profile: the vertical line corresponds to the B-scan (a). (d) is a non-planar reslice, defined by the curve drawn by the user in (c). The non-planar reslice shows an unrolled, frontal view of the spine, from neck (top) to coccyx (bottom). 